\title{
TRANSPLANTE HEPÁTICO EM TESTEMUNHAS DE JEOVÁ NO HOSPITAL SANTA ISABEL EM BLUMENAU - SANTA CATARINA: LEVANTAMENTO DE DADOS DE PRONTUÁRIOS
}

\section{Liver transplantation in Jehovah's Witnesses at Santa Isabel Hospital in Blumenau - Santa Catarina: Survey of data from medical records}

\author{
Marcelo Augusto Scheidemantel Nogara1, Gabriela Tiemi Hirosse², Vanessa Ferrari², \\ Mariana Viecelli Menezes da Silva²
}

\begin{abstract}
RESUMO
Objetivo: Realizar levantamento de dados sobre a ocorrência de transplantes hepáticos em Testemunhas de Jeová no Hospital Santa Isabel (HSI), município de Blumenau, Santa Catarina. Métodos: Trata-se de estudo observacional analítico transversal, e o critério de inclusão foi: pacientes de um grupo religioso específico (Testemunhas de Jeová), que por preceito religioso, não aceita transfusão sanguínea e transplantados hepáticos, de setembro de 2006 a junho de 2019. Estudo realizado pela análise de 22 prontuários, sendo 21 estudados devido à integralidade das informações, evidenciando dados epidemiológicos (idade, sexo, cidade de origem e patologia), escore MELD e necessidade de transfusão sanguínea intra e pós-operatória. Coleta realizada de agosto a outubro de 2019. Resultados: A média de idade dos transplantados foi de 53,54 anos, sendo o sexo masculino predominante $(61,9 \% ; n=13)$. A etiologia prevalente foi Cirrose hepática por Hepatite C (HCV) sem hepatocarcinoma $(38,9 \% ; n=8)$. Dos 21 pacientes estudados, nove são do estado catarinense $(40,9 \%)$ e 12 de outros seis estados brasileiros (57,14\%). Na avaliação MELD, 33,33\% $(n=7)$ dos pacientes foram classificados como menor que 15, 52,38\% ( $n=11)$ com MELD entre 15 e 25, e 14,28\% ( $n=3)$, maior que 25. Apenas três transplantados $(14,28 \%)$ necessitaram transfusão de elementos sanguíneos. Conclusão: Este trabalho demonstra que o serviço do HSI é uma referência nacional de transplante de órgãos, com número crescente de pacientes advindos de fora do estado catarinense. O procedimento demonstra também grande sucesso entre pacientes Testemunhas de Jeová, uma vez que dentre os 21 analisados, em apenas três houve necessidade de administração de algum tipo de hemoderivado.
\end{abstract}

Descritores: Transplante de Fígado; Testemunhas de Jeová; Insuficiência Hepática; Transfusão Sanguínea.

Instituições:

1 Serviço de Gastroenterologia, Hepatologia e Cirurgia Geral Equipe de Transplantes do Hospital Santa Isabel, Blumenau/SC, Brasil

2 Curso de Medicina da Universidade Regional de Blumenau FURB; Blumenau/SC, Brasil.

\section{Correspondência:}

Marcelo Augusto Scheidemantel Nogara

marcelonogara@gmail.com

https://doi.org/10.53855/bjt.v24i2.011

Recebido em 20/03/2021 Aceito em: 19/05/2021

\section{INTRODUÇÃO}

Segundo o Ministério da Saúde, o Brasil detém o maior sistema público de transplantes do mundo, e é o segundo em números absolutos de procedimentos, ${ }^{1} \mathrm{o}$ que torna o país referência mundial no assunto. Dados da Associação Brasileira de Transplantes de Órgãos (ABTO) revelam que dentre os transplantes de órgãos sólidos e tecidos, o hepático é o terceiro mais realizado no país e no estado de Santa Catarina, ${ }^{2}$ ficando atrás apenas dos transplantes de córnea e rim. A mesma base de dados ainda informa que no ano de 2018 foram realizados 135 procedimentos de transplantes de fígado em território catarinense; destes, 112 ocorreram no Hospital Santa Isabel em Blumenau. 
Desde 2002, quando foi realizado o primeiro transplante hepático no estado, milhares de pacientes passaram pela equipe do HSI e foram admitidos na fila de transplantes por diferentes doenças. ${ }^{3}$ Dentre as características de cada paciente, a religiosidade é uma particularidade que possui significativa importância; nesse ponto, destacase a questão das Testemunhas de Jeová (TJ).

Por razões religiosas e segundo o que interpretam na passagem bíblica em Levítico 17:13,14 ${ }^{4}$ ("Qualquer pessoa, filho de Israel ou estrangeiro residente entre vós, que caçar um animal ou ave que é permitido comer, deverá derramar seu sangue e recobri-lo com terra. Pois a vida de toda carne é o sangue. Por isso eu ordenei aos filhos de Israel: Não comerei o sangue de carne de alguma, pois a vida de toda carne é o sangue, e todo aquele que o comer será exterminado!"), as Testemunhas de Jeová recusam a transfusão de qualquer produto sanguíneo (glóbulos vermelhos, plaquetas ou plasma fresco congelado) que foi removido de um terceiro ou mesmo a autotransfusão sanguínea retirada de antemão e guardada, mas podem aceitar transplantes de órgãos sólidos como rins, pâncreas, coração, pulmão e fígado.

Sabe-se que o transplante hepático, em geral, necessita de transfusão sanguínea, por ser o maior órgão sólido do corpo humano e extremamente vascularizado; sendo assim, a recusa de hemoderivados dificulta o transplante.

O fundamento deste estudo está pautado no levantamento de dados de todos os prontuários de transplantes hepáticos já realizados em Testemunhas de Jeová no HSI desde o início do programa (setembro de 2006) até junho de 2019.

\section{PACIENTES E MÉTODOS}

O presente trabalho foi aprovado pelo Comitê de Ética da Fundação Universidade Regional de Blumenau (FURB) sob o número de Certificado de Apresentação para Apreciação Ética 13246619.6.0000.5370, isentando de Termo de Consentimento Livre e Esclarecido (TCLE) por já haver autorização, para fins de estudos médicos, em TCLE realizado no pré-transplante com os pacientes admitidos na instituição para esse determinado procedimento.

A pesquisa apresentou aumento no número de pacientes que estavam no projeto submetido ao Comitê de Ética, de 15 para 22. No entanto, um paciente precisou ser excluído do estudo por falta de informações suficientes nos prontuários.
O diagnóstico de insuficiência hepática nos pacientes TJ foi elaborado através de critérios clínicos (encefalopatia e/ou ascite; peritonite bacteriana espontânea recorrente; sangramento digestivo recidivante por hipertensão portal; fadiga ou desnutrição acentuadas; prurido intratável; síndrome hepatorrenal ou hepatopulmonar; doença óssea; colangites de repetição; neuropatia) e laboratoriais (níveis de bilirrubina e creatina séricas e INR), estando esses de acordo com a classificação da escala MELD (Model of End-Stage Liver Disease), cujo valor maior ou igual a 15 evidencia hepatopatia grave.

Trata-se de um estudo transversal observacional analítico, cuja abrangência está restrita a um grupo religioso específico. O estudo foi feito mediante a coleta de dados dos prontuários de todos os pacientes TJ transplantados no HSI, sendo avaliados quanto à necessidade ou não de transfusão sanguínea ou hemoderivados durante ou após o procedimento cirúrgico. Juntamente, foram coletados dados epidemiológicos (idade, sexo, procedência, MELD e etiologia dos transplantes).

\section{RESULTADOS}

Do ano de 20025 até junho de 2019 6, foram realizados no Brasil 25.003 transplantes hepáticos, sendo 1.342 pela equipe do Hospital Santa Isabel de Blumenau-SC,7 representando $8,79 \%$ do total do país.

Dentre todos os procedimentos realizados na cidade de Blumenau ( $n=1342), 22$ são de Testemunhas de Jeová $(1,63 \%)$. No entanto, a presente análise limita-se a apenas 21 casos, pois somente deles foram obtidas informações completas. (Tabela 1).

Nos números apresentados na tabela 1, o sexo masculino predomina, representando cerca de $61,9 \%$ dos casos $(n=13)$. Os transplantados possuem idade entre $33 \mathrm{e}$ 69 anos, com média de 53,54 anos e desvio padrão de $10,1 \%$. Quanto à procedência, 40,9\% $(n=9)$ são do estado de Santa Catarina, sendo quatro pacientes $(19,06 \%)$ da cidade de Blumenau, cinco $(23,8 \%)$ de outras cidades catarinenses e 12 de outros seis estados brasileiros (57,14\%).

Com relação à avaliação do MELD de admissão, sete $(33,33 \%)$ pacientes apresentaram MELD inferior a 15 , enquanto $11(52,38 \%)$ ficaram no intervalo de 15 a 25 e, apenas, $3(14,28 \%)$ acima de 25.

Quanto à etiologia, predominou cirrose hepática por hepatite $C$ sem hepatocarcinoma $(38,9 \% ; n=8)$. Outras patologias encontradas foram: Hepatite $\mathrm{C}$ concomitante 
a hepatocarcinoma - HCC $(23,80 \% ; n=5)$, Hepatite autoimune $(9,52 \% ; n=2)$, Cirrose criptogênica $(9,52 \%$; $\mathrm{n}=2)$, Doença policística de rim e fígado $(4,76 \%$; $\mathrm{n}=1)$, Esteato hepatite não alcoólica $(4,76 \% ; n=1)$, Esquistossomose $(4,76 \% ; n=1)$ e Colangite esclerosante primária $(4,76 \% ; n=1)$ (Tabela 2$)$.

$\mathrm{Na}$ maioria dos casos $(85,71 \%$; $\mathrm{n}=18)$, não houve necessidade de reposição de hemoderivados intra ou pós-operatório, sendo que apenas três $(14,28 \%)$ apresentaram tal necessidade.

Tabela 1 - Informações gerais coletadas nos prontuários

\begin{tabular}{lll}
\hline Dados Pessoais e de Identificação & N & $\%$ \\
\hline
\end{tabular}

\section{Gênero}

Masculino

13

61,9

Feminino

8

38,09

\section{Faixa Etária}

$$
\begin{aligned}
& 30-40 a \\
& 40-50 a \\
& 50-60 a \\
& >60 a
\end{aligned}
$$

3

14,28

19,04

33,33

33,33

\section{Procedência}

$\begin{array}{lcc}\text { SC } & 9 & 40,9 \\ \text { SP } & 5 & 22,72 \\ \text { RS } & 2 & 9,09 \\ \text { PR } & 2 & 9,09 \\ \text { ES } & 1 & 4,54 \\ \text { BA } & 1 & 4,54 \\ \text { MG } & 1 & 4,54\end{array}$

\begin{tabular}{|c|c|c|c|}
\hline Sim & & 3 & 14,28 \\
\hline Não & & 18 & 85,71 \\
\hline TOTAL & 21 & 100 & \\
\hline
\end{tabular}

\section{MELD}

$\begin{array}{lll}<15 & 7 & 33,33 \\ 15-25 & 11 & 52,38 \\ >25 & 13 & 14,28\end{array}$

\begin{tabular}{|c|c|c|}
\hline PATOLOGIA & $\mathbf{N}$ & $\%$ \\
\hline Hepatite C & 13 & 61,90 \\
\hline Com Hepatocarcinoma & 5 & 23,80 \\
\hline Sem Hepatocarcinoma & 8 & 39,09 \\
\hline Hepatite Autoimune & 2 & 9,52 \\
\hline Cirrose Criptogênica & 2 & 9,52 \\
\hline Doença Policística de rim e fígado & 1 & 4,76 \\
\hline Esteato Hepatite não alcoólica & 1 & 4,76 \\
\hline Esquistossomose & 1 & 4,76 \\
\hline Colangite Esclerosante Primária & 1 & 4,76 \\
\hline Total & 100 & \\
\hline
\end{tabular}

\section{Hemoderivado}

Tabela 2 - Patologias que levaram o paciente ao transplante hepático.

\section{DISCUSSÃO}

As transformações éticas, morais e legais associadas ao progresso técnico-científico dos últimos anos trouxeram situações novas às práticas em saúde, modificando o relacionamento dos profissionais com seus pacientes, de modo a torná-lo menos orientado aos deveres e obrigações morais (deontologia) e mais voltado ao respeito à autonomia e ao pluralismo moral. ${ }^{8}$ Os pontos de vista médico e jurídico nem sempre são concordantes, visto que os médicos estão condicionados a enxergar a manutenção da vida biológica como bem supremo, enquanto no outro discute-se sobre a autonomia, liberdade de consciência e o direito sobre o próprio corpo do paciente. ${ }^{9}$

O respeito mútuo às convicções pessoais, como a questão religiosa, trouxe à medicina alguns desafios diante dos entraves que cada doutrina apresenta a procedimentos médicos; a mais conhecida delas são as Testemunhas de Jeová, que têm em sua bíblia passagens que proíbem seus adeptos a receber transfusão sanguínea, pois acreditam que esse procedimento pode resultar na condenação eterna. Em seu Livro Sagrado, há passagens como: "Somente a carne com sua alma - seu sangue - não deveis comer" (Gênesis 9:3, 4); "não deveis tomar o sangue de carne alguma, pois a vida de toda carne é o seu sangue. Qualquer pessoa que tomar dele será cortada. Tens de derramar seu sangue e cobri-lo com pó" (Levíticos 17:13, 14). "Que se abstenham [...] da fornicação, e do estrangulamento, e do sangue" (Atos 15:19-21).4, ${ }^{10}$ No entanto, a proibição não é absoluta, eles permitem o 
uso de alguns componentes do sangue como albumina, imunoglobulinas e preparados para hemofílicos; além disso, a circulação extracorpórea, transplantes de órgãos e hemodiálise também são aceitas.

Deve-se lembrar que com relação ao conflito médicojurídico, não se trata dos direitos individuais do cidadão, mas o direito à liberdade religiosa e à obrigação e ao dever do Estado em garantir a saúde de todos. O direito à vida não se resume em mera existência carnal, mas da mesma forma à dignidade humana e seus valores morais, espirituais e psicológicos.

O Código de Ética Médica ${ }^{11}$ vigente apresenta nos capítulos I, II e IV (Princípios Fundamentais, Direitos dos Médicos e Direitos Humanos, respectivamente) artigos condizentes à temática da pesquisa. Em resumo, os artigos I, II, IV, VI, VII e XXI do capítulo I são discursos da profissão médica, a qual deve zelar pela saúde do ser humano sem qualquer tipo de discriminação com absoluto respeito, atuando com autonomia sempre em benefício do paciente e jamais contra sua dignidade e integridade. Em relação ao direito dos médicos, o artigo IX do segundo capítulo permite ao médico recusar-se a realizar atos médicos que, embora permitidos por lei, sejam contrários aos ditames de sua consciência, como por exemplo, recursar-se a realizar transplante hepático em um paciente que se nega a receber transfusão sanguínea. O último capítulo citado, por sua vez, traz nos artigos 22, 23 e 24 o veto de ações ao médico, como deixar de obter consentimento do paciente ou de seu representante legal, tratar o ser humano sem civilidade ou consideração, bem como deixar de garantir ao paciente o direito de livre escolha.

Caso a transfusão sanguínea seja um obstáculo intransponível à conformidade do recorrente à cirurgia tradicional, o Estado deve, obrigatoriamente, disponibilizar recursos para que o procedimento se dê por outra forma, quando no distrito não houver disponibilidade profissional ou ambiental (como em hospitais inaptos a determinados procedimentos).

A era dos transplantes de fígado em seres humanos é recente; iniciou em 1963, através do pioneirismo de T. E. Starzl. ${ }^{12} \mathrm{O}$ transplante hepático foi aceito como opção terapêutica eficaz para pacientes com o órgão em estágio terminal e insuficiência hepática aguda, apresentando um índice de sobrevivência global em três anos ao redor de $80 \% .^{13,14}$ É indubitável dizer que a postura firme das TJ em rechaçar transfusões sanguíneas alavancou 0 progresso científico de descoberta e aprimoramento de tratamentos alternativos. ${ }^{15}$ Além disso, foi criada uma rede de âmbito internacional de Comissões de Ligações com Hospitais $(\mathrm{COLIH})$, presente em 230 países, que assessoram a transferência de pacientes a equipes médicas que aceitam alternativas às transfusões de sangue, como é o caso do HSI. A partir da década de 90 começaram a surgir na literatura relatos de transplantes hepáticos ortotópico, sem uso de transfusão sanguínea ou hemoderivados. Esse procedimento passou a ser possível somente após refinamento cirúrgico e anestésico, e avanços na medicina intensiva, com diminuição da morbidade e mortalidade. ${ }^{16}$

Nomeada Capital Catarinense de Transplante de Órgãos, Blumenau localiza-se no segundo estado do país que mais doa, com taxas superiores a 40 doadores por milhão de população. ${ }^{17}$ Sancionada em setembro de 2019 pelo governador Carlos Moisés, a cidade recebeu reconhecimento devido ao grande número de procedimentos realizados, em especial no Hospital Santa Isabel. No período de 2011 a 2018, a instituição foi responsável por $832^{7}$ dos $967^{17}$ transplantes realizados no estado, perfazendo $86 \%$ do total.

O estudo em questão evidenciou a influência da cidade que atrai pacientes de diversas partes do estado e do país $(80 \% ; n=17)$, validando a eficiência e abrangência do serviço prestado. Quando comparado a uma pesquisa realizada nessa mesma instituição (HSI) no ano de 2006 sobre os primeiros transplantes no estado, 3 é notável o crescimento da representatividade da cidade no setor de transplantes a nível estadual, e principalmente nacional, uma vez que a taxa de procedência de pacientes de fora do estado não superou os $9 \%$ e no presente estudo alcançou $57,14 \%$.

Houve predominância do sexo masculino, dado concordante com outros estudos ${ }^{3,18,19}$ de transplantes na população em geral, não apenas em Testemunhas de Jeová. A promiscuidade sexual, o uso de drogas injetáveis em maior quantidade em comparação às mulheres, bem como o etilismo são explicações plausíveis para determinada predominância. ${ }^{20}$ No âmbito de idade dos transplantados, também obtivemos resultados semelhantes aos encontrados na população sem restrição religiosa; ${ }^{19}$ a média de idade foi de 53,54 anos, enquanto que no estudo comparado, foi de 50,8 anos. No entanto, o resultado divergiu de outro feito com grupo religioso $\mathrm{TJ},{ }^{21}$ no qual a idade média foi de 41 anos, ou seja, aproximadamente 12 anos a menos da idade média encontrada em nosso estudo. Apesar da discrepância entre as pesquisas, ainda assim, fica evidente que as hepatopatias em geral apresentam um curso arrastado de evolução. ${ }^{18}$

Quanto às patologias que indicaram o transplante, a hepatite $C$ foi a de maior índice dentre as sete causas encontradas em nossa e nas demais pesquisas realizadas anteriormente na mesma instituição. ${ }^{3,19}$ 
Dentre os 21 pacientes pesquisados, 18 deles $(85,71 \%)$ não necessitaram de qualquer tipo de hemoderivado. Em estudo menor, ${ }^{21}$ apesar de nenhum dos nove transplantados requererem hemoderivado, todos realizaram preparação prévia de aumento do hematócrito com eritropoietina, suplementação de ferro e ácido fólico.

É imperativo negar o desafio que é para o cirurgião realizar uma cirurgia de grande porte, como o transplante hepático, que exige extensa dissecção e envolvimento de importantes vasos, sem perdas volêmicas que possam comprometer o estado geral do paciente.

Todos os procedimentos do estudo vigente foram realizados sob a técnica de Piggyback, que segundo artigos de pesquisa, ${ }^{20}$ pode influenciar na menor necessidade de transfusão. Há muitos anos, na Medicina já se prioriza a prática de procedimentos com menor uso possível de hemoderivados integralmente entre os pacientes, optando-se por alternativas à transfusão. ${ }^{22}$ O periódico brasileiro Boletim, da Sociedade Brasileira de Hematologia e Hemoterapia ${ }^{23}$ aponta que no país, ainda ocorre a utilização indiscriminada de sangue e derivados, apesar dos enormes riscos inerentes à transfusão, o que mostra a necessidade de educação continuada em hemoterapia, com intuito de evitar transfusões que não são necessárias. Segundo o Dr. Michael Rose em entrevista para a revista Despertai, ${ }^{24}$ um paciente tratado sem sangue recebe tratamento cirúrgico da melhor forma possível. O Dr. Bruce Spiess, na mesma matéria, ${ }^{24}$ afirma duvidar da real repercussão positiva no paciente transfundido, inclusive, acreditando que em alguns casos, acabe prejudicando um melhor prognóstico.

Em relação à taxa de sobrevida imediata, até três meses de pós-transplante, dois pacientes vieram a óbito, sendo um deles por complicações de enxerto e outro por esquistossomose.

\section{CONCLUSÃO}

Este trabalho demonstrou que, apesar de se tratar de uma cirurgia de grande porte e extensa área de dissecção, é possível que o procedimento seja realizado sem necessidade de transfusão sanguínea. Um desafio à Medicina, tem se tornado tendência evitar em todos os procedimentos e pacientes, independentemente de possuir ou não impedimentos religiosos.

Os dados levantados por este estudo condizem com o encontrado na população em geral, na maioria dos critérios avaliados. A mesma proporção de concordância ocorre quando comparada aos estudos de transplante hepático em Testemunhas de Jeová, os quais são escassos e de menor casuística, dispondo o Hospital Santa Isabel da maior casuística do país (Brasil), denotando um serviço bem estabelecido nacional e internacionalmente, que recebe considerável número de pacientes de outras cidades e estados, onde não se faz transplante em Testemunhas de Jeová e se empenha ao máximo para respeitar as diferentes crenças e beneficiar a sociedade como um todo, sem distinção de cor, credo ou procedência, e com isso aumentar o conhecimento científico do serviço de transplant

\section{ABSTRACT}

Purpose: To collect data on the occurrence of liver transplants in Jehovah's Witnesses in Hospital Santa Isabel, Blumenau city, state of Santa Catarina. Methods: This is a cross-sectional observational study, which the inclusion criteria were patients from a specific religious group (Jehovah's Witnesses), who due to religious precept, do not accept blood transfusion and liver transplantation, from September 2006 to June. 2019. The study was conducted by analyzing 22 medical records, 21 of which were studied due to the completeness of the information, evidencing the epidemiological data (age, gender, city of origin and pathology), MELD score and need for intra and post-operative blood transfusion. Such collection was performed from August to October 2019. Results: The average age of the transplanted patients was of 53-54 years old, being the majority of male gender $(61.9 \%$; $n=13)$. The prevalent etiology was Hepatitis C (HCV) liver cirrhosis with no hepatocarcinoma (38.9\%; n=8). From the 21 patients studied, 9 are from Santa Catarina state (40.9\%), and 12 from six other Brazilian states (57.14\%). In the MELD assessment, 33.33\% $(n=7)$ of patients were classified as lower than 15, $52.38 \%(n=11)$ with MELD between 15 and 25, and $14.28 \%(n=3)$, higher than 25 . Only 3 transplanted (14.28\%) required blood element transfusion. Conclusion: This study demonstrates that the $\mathrm{HSI}$ service is a national reference for organ transplantation, with an increasing number of patients coming from other Brazilian states. Furthermore, the procedure shows great success among Jehovah's Witness patients, since among the 21 analyzed, only 3 required the use of a blood-derived product.

Keywords: Liver Transplantation; Jehovah's Witnesses; Liver failure; Blood transfusion 


\section{REFERÊNCIAS}

1. Ministério da Saúde [homepage na internet]. Doação de órgãos: transplantes, lista de espera e como ser doador [acesso em 28 out 2019]. Disponível em: http://www. saude.gov.br/saude-de- a-z/doacao-de-orgaos

2. Dimensionamento dos Transplantes no Brasil e em cada estado (2010-2017). Registro Brasileiro de Transplantes [Internet]. 2017 [cited 2019 Nov 6]; Available from: http:// www.abto.org.br/ abtov03/Upload/file/RBT/2017/rbtimprens a-leitura-compressed.pdf

3. Nogara MAS, et al. Avaliação dos transplantados hepáticos em Santa Catarina, de agosto de 2002 a julho 2004: relato dos primeiros 25 casos de um procedimento inédito no estado. Jornal Brasileiro de Transplantes. 2006 jan/mar; 9:474-477.

4. Bíblia online [Homepage na internet]. Levítico 17:14. [acesso em 28 out 2019]. Disponível em: https://www. bibliaonline. com.br/acf/lv/17/14

5. Registro Brasileiro de Transplantes [Internet]. São Paulo - SP: [publisher unknown]. No. 4, XV - [cited 2019 Sep 6]. Available from: http://www.abto.org.br/ abtov03/Upload/ file/RBT/2009/1.pdf
6. Registro Brasileiro de Transplantes [Internet]. São Paulo SP: [publisher unknown]. No. 2, XXV - [cited 2019 Sep 6]. Available from: http://www.abto.org.br/abt ov03/Upload/file/ RBT/2019/rbt2019-1sem-leitura.pdf.

7. Hospital Santa Isabel [Homepage na internet]. Transplantes (alta complexidade) [acesso em 28 out 2019]. Disponível em: http://www.santaisabel.com.br/sobre/1005/transplantes--altacomplexidade-

8. Azambuja LEO, Garrafa V. Testemunhas de jeová ante o uso de hemocomponentes e hemoderivados. Revista da Associação Médica Brasileira [Internet]. 2010 [cited 2019 Sep 6];56 DOI http://dx.doi.org/10.1590/ S0104-42302010000600022. Available from: http:// www.scielo.br/scielo.php?script=sci_arttext\&pid =S0104-42302010000600022.

9. Leiria CS. Transfusões de sangue contra a vontade de paciente da religião Testemunhas de Jeová.: Uma gravíssima violação de direitos humanos. Revista Prolegis [Internet].2009 Jun 29 [cited 2019 Oct 11]; Available from: https://prolegis. com.br/transfus \%C3\%B5es-de-sangue-contra-a-vontade-depaciente-da- religi $\% \mathrm{C} 3 \% \mathrm{~A} 30$-testemunhas-de-jeov\%C3\%A1uma-grav $\%$ C3\%ADssima- viola\%C3\%A7\%C3\%A3o-dosdireitos-humanos/. 